\title{
Covid-19 in patients with chronic lymphocytic leukemia: clinical outcome and B- and T-cell immunity during 13 months in consecutive patients
}

\author{
Lisa Blixt ${ }^{1,2}$, Gordana Bogdanovic ${ }^{3}$, Marcus Buggert ${ }^{4}$, Yu Gao ${ }^{4}$, Sophia Hober ${ }^{5}$, Katie Healy ${ }^{6}$, Hemming Johansson ${ }^{2}$, \\ Christian Kjellander ${ }^{7}$, Sara Mravinacova (1) ${ }^{5}$, Sandra Muschiol ${ }^{3}$, Peter Nilsson (D) ${ }^{5}$, Marzia Palma (D) ${ }^{1,2}$, Elisa Pin $^{5}$, C. I. Edvard Smith (D) ${ }^{8}$, \\ Olga Stromberg ${ }^{9}$, Margaret Sällberg Chen $\mathbb{D}^{6}{ }^{6}$, Rula Zain $\mathbb{D}^{8,10}$, Lotta Hansson $\mathbb{D}^{1,2,11 \bowtie}$ and Anders Österborg (iD) ${ }^{1,2,11}$
}

(C) The Author(s), under exclusive licence to Springer Nature Limited 2021

We studied clinical and immunological outcome of Covid-19 in consecutive CLL patients from a well-defined area during month 1-13 of the pandemic. Sixty patients (median age $71 \mathrm{y}$, range 43-97) were identified. Median CIRS was eight (4-20). Patients had indolent CLL $(n=38)$, had completed $(n=12)$ or ongoing therapy $(n=10)$. Forty-six patients $(77 \%)$ were hospitalized due to severe Covid-19 and 11 were admitted to ICU. Severe Covid-19 was equally distributed across subgroups irrespective of age, gender, BMI, CLL status except CIRS $(p<0.05)$. Fourteen patients $(23 \%)$ died; age $\geq 75$ y was the only significant risk factor $(p<0.05$, multivariate analysis with limited power). Comparing month $1-6$ vs $7-13$ of the pandemic, deaths were numerically reduced from $32 \%$ to $18 \%$, ICU admission from $37 \%$ to $15 \%$ whereas hospitalizations remained frequent ( $86 \%$ vs $71 \%)$. Seroconversion occurred in $33 / 40$ patients (82\%) and anti-SARS-CoV-2 antibodies were detectable at six and 12 months in 17/22 and 8/11 patients, respectively. Most (13/17) had neutralizing antibodies and 19/28 had antibodies in saliva. SARS-CoV-2-specific T-cells (ELISpot) were detected in 14/17 patients. Covid-19 continued to result in high admission even among consecutive and young early- stage CLL patients. A robust and durable B and/or T cell immunity was observed in most convalescents.

Leukemia (2022) 36:476-481; https://doi.org/10.1038/s41375-021-01424-w

\section{INTRODUCTION}

Patients with chronic lymphocytic leukemia (CLL) have an increased risk of severe infections due to disease- and treatment-related immunodeficiency $[1,2]$ that does not exclude novel precision therapeutics such as ibrutinib [3, 4]. Two early international surveys reported that hospital-admitted Covid-19 patients with CLL had a high fatality rate $[5,6]$. Understanding the full panorama of symptomatic Covid-19 infection in CLL requires analysis of consecutive patients from defined areas (i.e. without external referrals) identifying also those who were not admitted. This is particularly important since CLL patients may not respond sufficiently to Covid-19 vaccination $[7,8]$.

The present clinical immunological study included all CLL patients diagnosed with symptomatic Covid-19 during the first 13 months of the pandemic (March 2020-March 2021) in a well- defined geographical area (Stockholm, Sweden). Since diagnosis, follow-up and treatment of all CLL patients in our region is centralized to three hospitals and external referrals and office-based medicine are practically absent, we had a unique opportunity to identify all cases in order to generate reliable data on Covid-19 infection in the CLL population. We report here on their clinical outcome in two time periods of the pandemic (months 1-6 vs 7-13). In addition, we applied a panel of immune read-out methods to detect not only antibodies against SARS-CoV-2 but also their long-term persistence in convalescent serum. We investigated whether neutralizing antibody responses occurred (which may better correlate with protection) [9], if antibodies could be found in saliva and whether $\mathrm{T}$ cell immune response (IFN-gamma ELISpot assay) occurred in convalescents.

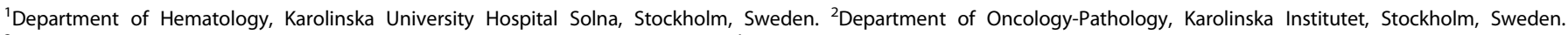

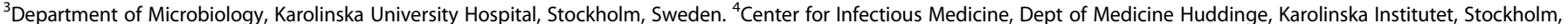

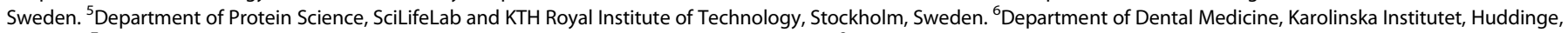

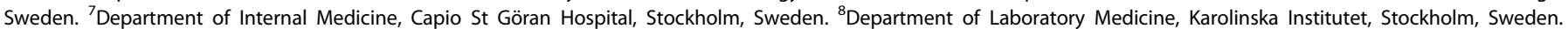

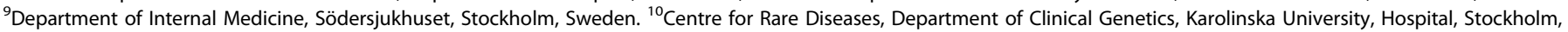
Sweden. ${ }^{11}$ These authors contributed equally: Lotta Hansson, Anders Österborg. ${ }^{凶}$ email: lotta.hansson@sll.se
} 


\section{MATERIALS AND METHODS}

\section{Patients and clinical assessments}

Patients with CLL at the three hematology centers in Stockholm (Karolinska University Hospital Solna, Capio St. Göran Hospital and Södersjukhuset, covering $>95 \%$ of all patients diagnosed with CLL in the region (as verified from the Information Network for Cancer Care (INCA) database) [10] (https://cancercentrum.se/samverkan/cancerdiagnoser/blod-lymfommyelom/kronisk-lymfatisk-leukemi-kll/statistik/) and who had a history of PCR-verified, symptomatic Covid-19 infection were included. The study period was set from March 1, 2020 until March 31, 2021. In the absence of a CLL Covid-19 database we formed a group consisting of our CLL physicians who systematically during the study period checked history of Covid-19 among all CLL patients at our 3 units and reported to our study coordinator. Baseline clinical characteristics were collected from patient files and included age, gender, Cumulative Illness Rating Scale (CIRS) [11], $\mathrm{BMI}$, smoking status, diabetes, CLL treatment history, blood counts and immunoglobulin (lg) levels. Data were collected on onset and type of Covid-19 infectious symptoms, hospitalization and its length, intensive care unit (ICU) admittance, treatment of Covid-19 as well as outcome, including long-term complications. The study was approved by the national ethics authority (www.etikprovningsmyndigheten.se). Written informed consent was obtained from each patient before samples were obtained for convalescent immune analyses (see below).

\section{Anti- SARS-CoV-2 antibody assays}

After onset of symptoms followed by a PCR-confirmed diagnosis of Covid19, convalescence blood samples were obtained at 3 months (in some cases 6 months). Samples were repeated at 6 and 12 months as available. Final assessment was performed before start of Covid-19 vaccination. Serum samples were routinely analysed for SARS-CoV-2 antibodies at the Dept. of Microbiology, Karolinska University Hospital, by different tests under the study period including SARS-CoV2-IgG test iFlash 1800 YHLO (CLIA) and Elecsys ${ }^{\circledR}$ Anti-SARS-CoV-2 (ECLIA, qualitative) and Elecsys ${ }^{\circledR}$ AntiSARS-CoV-2 S (ECLIA, quantitative) (Roche Diagnostics). YHLO determines the antibodies against SARS-CoV-2 nucleocapsid and spike (S) antigen, Roche qualitative against nucleocapsid antigen and Roche quantitative against spike antigen receptor binding site (RBD). The majority of samples at the six months and 12 months evaluation were analysed by Roche quantitative antibody test. Measurement range is $0.40-250 \mathrm{U} / \mathrm{mL}$ and values $<0.80 \mathrm{U} / \mathrm{mL}$ were considered negative [12]

In addition, neutralizing antibodies were analysed in a subset of patients using a competitive bead-based binding assay. Briefly, plasma samples were incubated (1/10 dilution) with biotinylated spike-f $(1 \mu \mathrm{g} / \mathrm{mL}$ spike-f, 1xPBS, $3 \%(\mathrm{w} / \mathrm{v})$ bovine serum albumin, $5 \%(\mathrm{w} / \mathrm{v})$ non-fat milk and $0.05 \%$ (v/v) Tween 20) for $1 \mathrm{~h}$ at room temperature (RT). Following this, the sample-antigen mix was transferred to a microtiter plate containing the ACE2 coupled beads and incubated $1 \mathrm{~h}$ at RT. After washing, the beads were incubated with $0.2 \%$ paraformaldehyde for 10 min at RT to crosslink the receptor-antigen complexes. The fluorescence intensity was measured on a FlexMap 3D instrument (Luminex corp.) by utilizing a Streptavidin-Rphycoerythrine conjugate. This method has been validated against a microneutralising, cell-based assay, using 169 negative and 133 positive samples. By that it has been shown that utilizing a cut-off based on three times the standard deviation of the mean of the negative samples, no false positive classification was made among the 169 negative samples. Among the 133 samples classified as positive in the cell-based microneutralising assay, 132 clearly inhibit the binding between spike-f and ACE2 and one was reported as a borderline sample. Samples with fluorescent signals above 1500 in the $1 / 10$ dilution are scored as negative. Samples with signals below 1500 in the 1/10 dilution and above 1000 in the 1/50 dilution are scored as + and samples with signals below 1000 and above 500 in the $1 / 50$ dilution are scored as ++ . Finally, samples with signals below 500 in the $1 / 50$ dilution are scored as +++ .

We also analysed SARS-CoV-2 antibodies in saliva using a self-sampling technique as recently described by Alkharaan et al [13]. Briefly, expectorated unstimulated whole saliva was collected using standardized self-collection kit with instruction and sample tubes provided by the study coordinator. The samples were sent in overnight and processed the same day for $-80^{\circ} \mathrm{C}$ storage. A multiplex bead-based serology assay was used for detection of $\mathrm{lgG}$ reactivities to 3 SARS-CoV-2 antigens (Spike S1S2 trimer, S1, and the C-terminal part of the nucleocapsid) in saliva samples, with internal controls of pre-pandemic $(n=12)$ as well as convalescence $(n=4)$ saliva samples in each run. Saliva antibody reactivity is expressed as median fluorescence intensity (MFI) and the assay cut-off for each antigen was determined using the mean $+6 x$ SD of the 12 negative pre-pandemic samples as previously described [13].

\section{SARS-CoV-2 specific T cells}

T-cells specific for SARS-CoV-2 were analysed by IFN-gamma ELISpot assay as recently described [14]. Briefly, PBMC (2.5 x 10E5/well) were seeded in filter plates precoated with anti-IFN-gamma and supplemented with overlapping peptides spanning the immunogenic domains of the SARSCoV-2 spike, membrane(M), nucleocapsid (N) and envelope (E) proteins, or control (Staphylococcal enterotoxin B (SEB)) peptides. Assays were incubated in duplicate for $24 \mathrm{~h}$ at $37^{\circ} \mathrm{C}$, washed and biotinylated antiIFN-gamma was added and developed by addition of avidin and substrate. Spot-forming units (SFUs) were counted using an automated ELISpot reader system as previously described. Data are presented as number of spots/well, as \% of CD3 T cells and as total numbers (IFN-gamma producing cells/uL). A patient was considered positive if $>20$ SFUs with $S$ and/or the $\mathrm{M}+\mathrm{N}+\mathrm{E}$ pool were recorded after background subtraction [14].

\section{Statistical Analysis}

All variables and analyses were pre-specified and selected from previous Covid-19 reports on large patient materials and mainly the $2 \mathrm{CLL}$ publications $[5,6]$.

Data is summarized using descriptive statistics such as counts, percentages, medians and range. Categorical variables were expressed as cross-tabulations and distributional differences were tested using the Chi-squared test. Univariate and multivariate modelling of the outcome death (within three months) and hospitalization (yes, no) were performed using logistic regression. Variables included in the univariate analysis were sex, age ( $\geq 75$ vs $<75$ ) as in [5], BMI ( $\geq 25$ vs $<25$ ), diabetes (yes, no), CIRS ( $>8$ vs $\leq 8$ ) based on our median, CLL treatment (never vs ever treated) as in [5], smoking status (never vs ever smoker) and hypogammaglobulinemia. Variables without missing data were included in the multivariate analysis of both outcomes. Results from these models are reported as odds-ratios (OR) together with $95 \%$ confidence intervals $(\mathrm{Cl})$ and Wald $p$ values. The fit of the multivariate models was evaluated using Pearson goodness-of-fit test. Stata version 17 was used for the statistical analysis. A numerical comparison of clinical outcome between month 1-6 vs 7-12 of the pandemic was also performed.

\section{RESULTS}

\section{Baseline characteristics}

During the first year of the pandemic (March 2020 - March 2021) we identified 60 patients with PCR-verified, symptomatic Covid-19 and CLL in our geographical region (Stockholm, Sweden). None had been vaccinated against SARS-CoV-2. Preliminary data from seven patients included in this study were previously reported in an international survey [5]. Five patients with previously unknown CLL were included as they were admitted due to severe Covid-19.

Baseline characteristics are shown in Table 1. The median age was 71 years (range 43-97) and 65\% were men. Median CIRS was eight (range 4-20) and median BMl was 25 (range 19-42). Diabetes was present in $30 \%$ and $53 \%$ were never-smokers; only $3 \%$ were current smokers. Thirty-eight patients $(63 \%)$ had indolent, untreated CLL, $12(20 \%)$ had received prior anti-CLL therapy (all $>12$ months ago) and 10 patients (17\%) had ongoing therapy (Bruton's tyrosine kinase (BTK) inhibitor in 7 patients, chemoimmunotherapy in 1 , and venetoclax $+/-$ CD20 mAb in two patients) at the time of infection. Only $12 \%$ of the patients had progressive CLL and 2 patients received prophylactic (IV or SC) gammaglobulin therapy.

\section{Clinical outcome of Covid-19 infection}

Outcome results are summarized in Tables 1 and 2. Forty-six patients (77\%) were hospitalized due to severe Covid-19 and 11 of them (24\%) were admitted to ICU. The median length of hospitalization was 11 days (range 1-38) and at ICU 10 days (range 4-21). Severe Covid-19 was equally distributed across patient subgroups irrespective of age, gender, BMI, CLL status, 
Table 1. Clinical characteristics in relation to hospitalization, ICU admission and death.

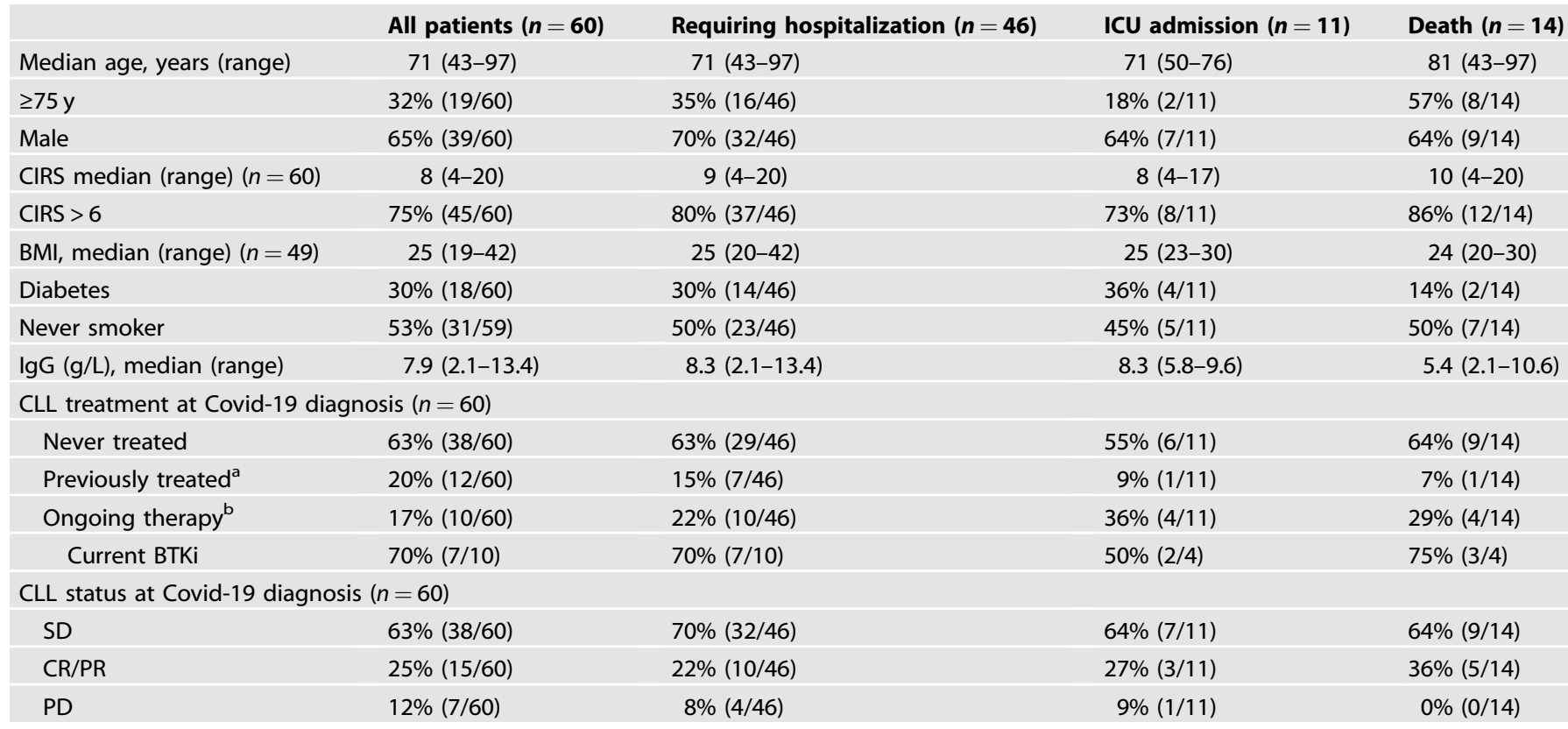

${ }^{\mathrm{a} A l l}>12$ months ago.

one patient received venetoclax; one patient received venetoclax + rituximab; one patient received bendamustin + rituximab; seven patients received BTKi.

Table 2. Outcome and management of Covid-19 among CLL patients $(n=60)$ in two time periods.

\begin{tabular}{|c|c|c|c|}
\hline & All patients & Admitted month 1-6 of the pandemic & Admitted month $7-13$ of the pandemic \\
\hline Hospital admission & $77 \%(46 / 60)$ & $86 \%(19 / 22)$ & $71 \%(27 / 38)$ \\
\hline ICU admission & $24 \%(11 / 46)$ & $37 \%(7 / 19)$ & $15 \%(4 / 27)$ \\
\hline Death (if admitted) & $28 \%(13 / 46)^{c}$ & $32 \%(6 / 19)^{c}$ & $26 \%(7 / 27)^{c}$ \\
\hline \multicolumn{4}{|l|}{ Intervention } \\
\hline Supplemental oxygen & $85 \%(39 / 46)$ & $84 \%(16 / 19)$ & $85 \%(23 / 27)$ \\
\hline Non-invasive ventilation (incl $\mathrm{HFNO}^{\mathrm{a}}$ ) & $33 \%(15 / 46)$ & $37 \%(7 / 19)$ & $30 \%(8 / 27)$ \\
\hline Mechanical ventilation & $13 \%(6 / 46)$ & $32 \%(6 / 19)$ & $0 \%(0 / 27)$ \\
\hline IV vasopressors & $18 \%(8 / 45)$ & $32 \%(6 / 19)$ & $8 \%(2 / 26)$ \\
\hline Hemodialysis & $9 \%(4 / 46)$ & $11 \%(2 / 19)$ & $7 \%(2 / 27)$ \\
\hline \multicolumn{4}{|l|}{ Agents used for Covid-19 } \\
\hline Hydroxychloroquine & $4 \%(2 / 45)$ & $11 \%(2 / 19)$ & $0 \%(0 / 26)$ \\
\hline Remdesivir & $26 \%(12 / 46)$ & $5 \%(1 / 19)$ & $41 \%(11 / 27)$ \\
\hline Tocilizumab & $2 \%(1 / 45)$ & $5 \%(1 / 19)$ & $0 \%(0 / 26)$ \\
\hline$I V I G^{b}$ & $4 \%(2 / 45)$ & $0 \%(0 / 19)$ & $8 \%(2 / 26)$ \\
\hline Corticosteroids & $65 \%(30 / 46)$ & $47 \%(9 / 19)$ & $78 \%(21 / 27)$ \\
\hline Convalescent plasma & $7 \%(3 / 46)$ & $5 \%(1 / 19)$ & $7 \%(2 / 27)$ \\
\hline Anticoagulation & $89 \%(41 / 46)$ & $84 \%(16 / 19)$ & $93 \%(25 / 27)$ \\
\hline
\end{tabular}

smoking status, diabetes and IgG level. CIRS (> 8 vs $\leq 8$ ) was significantly associated with hospitalization in univariate and multivariate analysis (OR $=4.9,95 \% \mathrm{Cl}: 1.0-24.1, p=0.048$ ) (Suppl. Table S1). Death occurred in $14 / 60$ patients (23\%) and in $13 / 46$ $(28 \%)$ of those who had been hospitalized. Age $\geq 75$ (vs $<75$ ) years was significantly associated with death both in univariate and multivariate analysis ( $\mathrm{OR}=4.3,95 \% \mathrm{Cl}: 1.0-18.3, p=0.049)$ (Suppl. Table S2).
No other pre-specified subgroup analysis reached statistical significance but our statistical power was limited $(n=60)$.

Five patients had previously unknown CLL and may have caused a bias in the analysis. When re-analysing outcome on the remaining 55 patients the survival effect of old age remained significant in the univariate analysis $(p=0.04)$ but was no longer significant in the multivariate analysis $(p=0.07)$. The impact of CIRS on hospitalization was no longer significant in either analysis 
(both $p=0.07)$. Forty-two of the 55 patients (76\%) were hospitalized and 10 of them (24\%) were admitted to ICU. Death occurred in $14 / 55$ patients (25\%).

Next, we compared numerically the risk of hospitalization, ICU admission and death, respectively, during the first 6 months of the pandemic vs month 7-13 (Table 2). Hospitalization due to severe Covid-19 was only slightly reduced in the second time period (from $86 \%$ to $71 \%$ ) whereas ICU admissions among hospitalized patients were numerically reduced from $37 \%(7 / 19)$ to $15 \%(4 / 27)$. Almost all our patients were admitted as needed and received active therapy against the infection and its complications. One patient deferred intensive care and opted for palliation due to co-morbidities and advanced age. While overall death rates were numerically reduced from $32 \%(7 / 22)$ to $18 \%(7 / 38)$, fatalities among hospitalized patients were less reduced (from $32 \%(6 / 19)$ in period 1 to $26 \%(7 / 27)$ in period 2) but the number of events was limited and did not allow statistical comparisons. Details on Covid-19 treatment in time period 1 and 2 are depicted in Table 2. Supplemental oxygen was used in $85 \%$ confirming severe Covid-19 in admitted patients. In the second time period there was a numerical increase in the use of remdesivir (from $5 \%$ to $41 \%$ ) but particularly corticosteroids became widely applied (from $47 \%$ to $78 \%$ ). BTK inhibitor therapy was held in most patients during the infection. No long-term, post-covid complications were reported in surviving patients at 3, 6 or 12 months of follow-up.

\section{Immunological results}

Individual results are depicted in Table 3. Forty convalescent patients were tested at least once (month three and onwards) for antibodies against SARS-CoV-2 and $33(82 \%)$ seroconverted in the sample taken at three or six months (Table 3). No baseline factor was significantly associated with seroconversion but the number of seronegative convalescents was low $(n=7)$. Anti- SARS-CoV-2 antibodies were detectable in most patients tested at 6 months (17/22 patients) and at 12 months (8/11 patients) of follow-up.

We then performed in-depth immune analyses in some of the convalescents. The results are summarized in Table 3. First, a confirmatory serology test (IgG antibodies against Spike RBD and Core proteins) confirmed the results of screening assay (not shown). We thereafter applied a pseudoneutralizing assay and found that the antibodies from 13 of 17 patients had neutralizing capacity (Table 3), of which one patient was classified as seronegative in conventional serology. Next we analysed saliva (self-sampling kit) for the presence of anti- SARS-CoV-2 antibodies [13]. Twenty-eight patients were included in this analysis which was performed after median time of 6 months (range: $2-13$ ); 19/28 (68\%) had detectable salivary antibodies specific to the S1S2 spike trimer while 13 of them had salivary antibodies to the nucleocapsid (Suppl. Table S3). One patient (010) had antibodies in saliva without seroconversion. Finally we analysed $\mathrm{T}$ cell immunity against SARS-CoV-2 using a validated IFN-gamma ELISpot assay [14]. Among the 17 patients tested, 14 (82\%) were positive (Table 3). The proportion and total number of SARS-CoV-2 specific $T$ cells varied considerably between individuals. A positive T cell assay was generally paralleled by seroconversion which in some tested individuals was associated also with antibodies in saliva (triple positive patients) (Table 3). One patient (010) had strongly positive ELISpot results, negative blood serology tests but had antibodies in saliva.

\section{DISCUSSION}

Due to the combined effect of disease- and treatment-related immunodeficiency, patients with hematological malignancies may be at high risk for developing severe disease if infected by SARS-CoV-2 $[15,16]$. Patients with CLL may be particularly vulnerable as indicated by two early international surveys reporting that more than one third of CLL patients had a fatal outcome if hospitalized due to Covid-19 $[5,6]$. However, these reports included patients from various sources and were identified in the very beginning of the pandemic. Less severe cases were not included. To get a full picture of the Covid-19 panorama in patients with CLL, we conducted a thorough analysis of all patients with CLL who were diagnosed with Covid-19 in a welldefined geographical region (Stockholm, Sweden) where external referrals and office based-medicine are absent. Repeated check-up of the INCA database has verified that more than $95 \%$ of all CLL patients in our region are referred to/followed at our three hematology units. Thus, we were able to identify practically all symptomatic CLL patients who tested positive for SARS-CoV-2 RNA in our region, thereby getting as close as possible to reflect a real-world panorama of the incidence and outcome of symptomatic Covid-19 in patients with CLL.

Given the limitations mentioned above, we found that as many as $77 \%$ of consecutive CLL patients diagnosed with Covid-19 developed severe disease requiring hospitalization, and of these $28 \%$ died from Covid-19 and its complications. Thus, the risk of death, if hospitalized, was similar to the results shown in the early international surveys $[5,6]$. We confirm that the death rate was significantly higher in patients 75 years or older [5]. Importantly, some patients were diagnosed with CLL as they were hospitalized due to severe Covid-19 infection. Including 5 patients with previously unknown CLL may represent a potential bias in our statistical analysis. The survival effect of old age remained significant in the univariate but not the multivariate analysis when these five patients were excluded. Another limitation of our study is that patients with mild Covid-19 and yet not detected/ unknown CLL may have been missed as blood counts may not have been regularly checked in routine health care among otherwise healthy individuals with mild Covid-19. The number of patients $(n=60)$ included provided limited power for multiple subgroup analyses. However, severe Covid-19 requiring hospitalization appeared to be similarly distributed across pre-defined subgroups irrespective of age, gender, BMI, diabetes, smoking, CLL status and hypogammaglobulinemia; comorbidities (CIRS) was the only significant risk factor. Of note, most patients diagnosed with Covid-19 were subject to hospital admission also during month 7-13 of the pandemic. ICU admittance and overall death rates declined numerically during the study period but the size of our study $(n=60)$ did not allow a statistical comparison between the two time periods. Another potential limitation of our study is that the degree of aggressiveness in fighting the infection and its complications may have varied. However, palliation was applied in one patient only and the general level of active anti-Covid therapy was high in our region. Thus, a potential between patient variation in the intensity of anti-covid therapeutic efforts is unlikely to explain the differences observed over time.

The second aim of our study was to conduct an in-depth analysis of SARS-CoV-2 immunity and its long-term persistence in CLL convalescents. Since there is limited knowledge about post-covid immunity in CLL patients, we performed repeated testing of antibodies against SARS-CoV-2 in our cohort and in-depth immune analysis of some patients. More than $80 \%$ of our 40 tested convalescent patients had seroconverted, which is in line with the $67 \%$ seroconversion reported recently among 21 CLL patients [17]. In otherwise healthy (non-CLL) convalescents anti-Spike in plasma may decay rapidly [18]. Data from our longitudinal follow-up revealed that most tested patients were seropositive at 6 and even 12 months of follow-up. Furthermore, the antibodies detected in our patients had neutralizing capacity, which is assumed to correlate with protection [9]. By applying self-collecting saliva [13] we found that around two third of tested patients had detectable antibodies against spike in saliva. Mucosal antibodies secreted into saliva are thought to be important as it has been recently shown that the oral cavity and saliva are virus replication sites that actively participates in SARS-CoV2 transmission [19]. Here, the frequency of saliva antibody-positive CLL patients is slightly lower than we recently reported for mild Covid-19 patients in a general population, in which specific salivary 
Table 3. Immunological results in Covid-19 convalescent CLL patients.

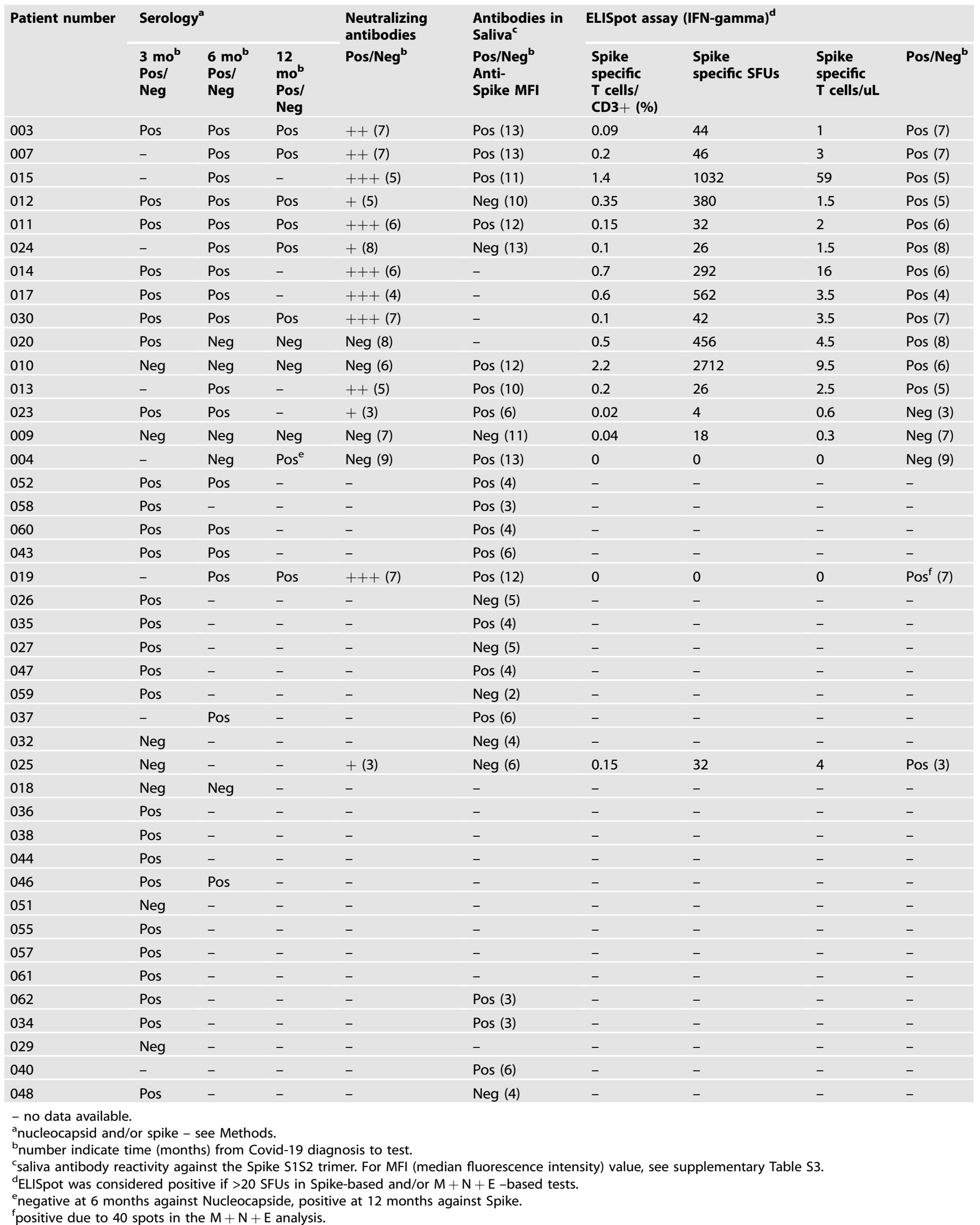


antibodies persisted in $88 \%$ (spike trimer) and $56 \%$ (capsid) in 9 months convalescence saliva [13]. In addition, we show that SARSCoV-2 specific T cell immunity occurs in the majority of CLL convalescents which was usually but not always paralleled by seroconversion. This is in line with a recent report on 5 patients with antibody deficiencies [20] and a group of 22 patients with mixed hematological malignancies [15]. Whether $T$ cell responses may occur also following Covid-19 vaccination in CLL is under investigation. Of note, $T$ cell responses in healthy individuals were often more robust after vaccination than in convalescent patients [21]. A limitation of our immune analyses is that not all tests were performed and repeated in all patients. It appears however that durable responses including neutralizing antibodies occur in most CLL patients and that some of them also have antibodies in saliva and/or T cell immunity i.e. some patients were positive in two or three assays whereas others were positive in only one test. In a series of UK healthcare workers with mild or asymptomatic SARS-CoV-2 infection discordant neutralizing antibody and $\mathrm{T}$ cell responses were observed [22]. This demonstrates the complexity of the immune response to this virus making it challenging to compare immunity between infected patient groups. However, the humoral and cellular response pattern observed among CLL patients does not seem to differ significantly as compared to a population not affected by CLL, even though the magnitude appears to be lower than in otherwise healthy convalescents. Collectively, our immune results indicate that robust and durable $B$ and/or T cell immunity may occur in most CLL patients following Covid-19 infection. Whether there is a common denominator for those few convalescent CLL patients who are completely negative in SARS-CoV-2 immune tests remains to be established. Our data suggest that the rate of seroconversion appears to be higher following natural infection in CLL patients than after Covid-19 vaccination [7, 8]. Patients who died from Covid-19 could not be included in the immune analysis which may at least partly explain why our rates on seroconversion appears higher than after de novo vaccination [7].

Whether convalescent CLL patients will benefit from booster vaccination is subject to an ongoing study.

\section{REFERENCES}

1. Langerbeins $P$, Eichhorst $B$. Immune dysfunction in patients with chronic lymphocytic leukemia and challenges during COVID-19 pandemic. Acta Haematol. 2021;144:508-18.

2. Palma M, Gentilcore G, Heimersson K, Mozaffari F, Nasman-Glaser B, Young E, et al. T cells in chronic lymphocytic leukemia display dysregulated expression of immune checkpoints and activation markers. Haematologica. 2017;102:562-72.

3. Mulder TA, Pena-Perez L, Berglof A, Meinke S, Estupinan HY, Heimersson $K$, et al. Ibrutinib has time-dependent on- and off-target effects on plasma biomarkers and immune cells in chronic lymphocytic leukemia. Hemasphere. 2021;5:e564.

4. Rogers KA, Mousa L, Zhao Q, Bhat SA, Byrd JC, El Boghdadly Z, et al. Incidence of opportunistic infections during ibrutinib treatment for B-cell malignancies. Leukemia. 2019:0:2527-30.

5. Mato AR, Roeker LE, Lamanna N, Allan JN, Leslie L, Pagel JM, et al. Outcomes of COVID-19 in patients with CLL: a multicenter international experience. Blood. 2020;136:1134-43.

6. Scarfo L, Chatzikonstantinou T, Rigolin GM, Quaresmini G, Motta M, Vitale C, et al. COVID-19 severity and mortality in patients with chronic lymphocytic leukemia: a joint study by ERIC, the European Research Initiative on CLL, and CLL Campus. Leukemia. 2020;34:2354-63.

7. Herishanu Y, Avivi I, Aharon A, Shefer G, Levi S, Bronstein Y, et al. Efficacy of the BNT162b2 mRNA COVID-19 vaccine in patients with chronic lymphocytic leukemia. Blood. 2021;137:3165-73.

8. Roeker LE, Knorr DA, Thompson MC, Nivar M, Lebowitz S, Peters N, et al. COVID19 vaccine efficacy in patients with chronic lymphocytic leukemia. Leukemia. 2021;35:2703-5.

9. Khoury DS, Wheatley AK, Ramuta MD, Reynaldi A, Cromer D, Subbarao K, et al. Measuring immunity to SARS-CoV-2 infection: comparing assays and animal models. Nat Rev Immunol. 2020;20:727-38.

10. Kimby EAM, Mattsson M. First report from the Swedish CLL-registry: a nationwide registry with high coverage (Abstract XVI iwCLL Sydney). Leuk Lymphoma. 2015;56:42.
11. Parmelee PA, Thuras PD, Katz IR, Lawton MP. Validation of the Cumulative Illness Rating Scale in a geriatric residential population. J Am Geriatr Soc. 1995;43:130-7.

12. Higgins V, Fabros A, Kulasingam V. Quantitative measurement of Anti-SARS-CoV-2 antibodies: analytical and clinical evaluation. J Clin Microbiol. 2021;59:e03149-20.

13. Alkharaan $\mathrm{H}$, Bayati $\mathrm{S}$, Hellstrom $\mathrm{C}$, Aleman S, Olsson A, Lindahl K, et al. Persisting Salivary IgG against SARS-CoV-2 at 9 Months After Mild COVID-19: A Complementary Approach to Population Surveys. J Infect Dis. 2021;224:407-14.

14. Sekine T, Perez-Potti A, Rivera-Ballesteros O, Stralin K, Gorin JB, Olsson A, et al. Robust $\mathrm{T}$ cell immunity in convalescent individuals with asymptomatic or mild COVID-19. Cell. 2020;183:158-168 e114.

15. Bange EM, Han NA, Wileyto P, Kim JY, Gouma S, Robinson J, et al. CD8(+) T cells contribute to survival in patients with COVID-19 and hematologic cancer. Nat Med. 2021;27:1280-9.

16. Vijenthira A, Gong IY, Fox TA, Booth S, Cook G, Fattizzo B, et al. Outcomes of patients with hematologic malignancies and COVID-19: a systematic review and meta-analysis of 3377 patients. Blood. 2020;136:2881-92.

17. Roeker LE, Knorr DA, Pessin MS, Ramanathan LV, Thompson MC, Leslie LA, et al. Anti-SARS-CoV-2 antibody response in patients with chronic lymphocytic leukemia. Leukemia. 2020;34:3047-9.

18. Anand SP, Prevost J, Nayrac M, Beaudoin-Bussieres G, Benlarbi M, Gasser R, et al. Longitudinal analysis of humoral immunity against SARS-CoV-2 Spike in convalescent individuals up to eight months post-symptom onset. Cell Rep. Med. 2021;2:100290.

19. Huang $\mathrm{N}$, Perez $\mathrm{P}$, Kato T, Mikami $\mathrm{Y}$, Okuda K, Gilmore RC, et al. SARS-CoV-2 infection of the oral cavity and saliva. Nat Med. 2021;27:892-903.

20. Kinoshita $H$, Durkee-Shock J, Jensen-Wachspress $M$, Kankate VV, Lang $H$, Lazarski CA, et al. Robust antibody and T cell responses to SARS-CoV-2 in patients with antibody deficiency. J Clin Immunol. 2021;41:1146-53.

21. Gallagher KME, Leick MB, Larson RC, Berger TR, Katsis K, Yam JY, et al. SARS -CoV2 T-cell immunity to variants of concern following vaccination. bioRxiv. 2021.

22. Reynolds CJ, Swadling L, Gibbons JM, Pade C, Jensen MP, Diniz MO, et al. Discordant neutralizing antibody and $T$ cell responses in asymptomatic and mild SARS-CoV-2 infection. Sci Immunol. 2020;5:eabf3698.

\section{ACKNOWLEDGEMENTS}

We thank the patients who donated blood and saliva in this study. The technical assistance of Cecilia Hellström, Kia Heimersson, Jennie Olofsson, Eni Andersson, Lovisa Skoglund, Jamil Yousef, Anna Fahlén and Leila Relander is highly appreciated. Supported by grants from Swedish Blood Cancer Society, Swedish Cancer Foundation, Swedish Research Council, Erling Persson Family Foundation, Gilead Fellowship Award, Gunnar Grimfors Foundation, Knut and Alice Wallenberg Foundation and Region Stockholm.

\section{AUTHOR CONTRIBUTIONS}

LB, LH and AÖ designed the study, identified and met patients, analysed the data and wrote the manuscript. GB and SMu analysed and interpreted results and edited the manuscript. $\mathrm{HJ}$ was the responsible statistician and performed statistical analyses and edited the manuscript. $\mathrm{MB}$ and $\mathrm{YG}$ analysed data, interpreted results and edited the manuscript. SH, MSC, KH, SMr, PN and EP analysed and interpreted results and edited the manuscript. OS and CK identified and met patients and approved the manuscript. MP identified and met patients and edited the manuscript. CIES and RZ were involved in the planning of the study, interpretation of data and edited the manuscript.

\section{COMPETING INTERESTS}

The authors declare no competing financial interests. MB is a consultant for Oxford Immunotech.

\section{ADDITIONAL INFORMATION}

Supplementary information The online version contains supplementary material available at https://doi.org/10.1038/s41375-021-01424-w.

Correspondence and requests for materials should be addressed to Lotta Hansson.

Reprints and permission information is available at http://www.nature.com/ reprints

Publisher's note Springer Nature remains neutral with regard to jurisdictional claims in published maps and institutional affiliations. 